\title{
A case of recurrent Mikulicz's disease with mononeuritis multiplex
}

\author{
ATSUSHI MIZUMA, MAIKO KOUCHI, CHIKAGE KIJIMA, SACHIKO YUTANI, \\ TSUYOSHI UESUGI, EIICHIRO NAGATA, SHUNYA TAKIZAWA
}

Department of Neurology, Tokai University School of Medicine, Isehara, Japan

\begin{abstract}
We report an 82-year-old man with recurrence of Mikulicz's disease accompanied with mononeuritis multiplex. On admission, both upper eyelids, the salivary gland, the dorsum of the left hand and both legs were swollen. Neurological examination showed motor weakness of distal limbs (manual muscle testing 3/5) and decreased touch, pain and vibration sensation of the dorsum of the left hand and both legs. Deep tendon reflex in both legs was also decreased. We diagnosed Mikulicz's disease based on high serum immunoglobulin ( $\mathrm{Ig}) \mathrm{G} 4(630 \mathrm{mg} / \mathrm{dl}$, 26.1\% of total $\mathrm{IgG})$ and lacrimal gland biopsy findings. Clinical symptoms and motor conduction study findings improved after steroid therapy. However, tapering of the steroid dose resulted in recurrence two years later. Steroid therapy is usually effective for IgG4-related neuropathy, and we found that an increase of steroid dose was effective to treat the recurrence. But, in general, a suitable maintenance dose of steroid in combination with an immunosuppressant may be necessary to prevent relapse.
\end{abstract}

Key words: Mikulicz's disease, mononeuritis multiplex, IgG4, steroid therapy.

(Centr Eur J Immunol 2018; 43 (4): 490-494)

\section{Introduction}

Immunoglobulin (Ig)G4-related disease (IgG4RD) is proposed as a novel disease entity associated with polyclonal increase and infiltration of IgG4 [1-3]. Pathologically, IgG4 plasma cells account for more than $40 \%$ of total IgG plasma cells in patients [4]. Single or multiple organs may be affected, with various symptoms, such as pancreatitis and sclerosing cholangitis [5]. IgG4RD, which responds well to steroid therapy [6], is known to be associated with hypertrophic pachymeningitis and hypophysitis in the central nerve system [7,8]. On the other hand, there are few reports of peripheral nerve palsy associated with IgG4RD [9] and neuropathological criteria for IgG4RD have not been established.

Mikulicz's disease (MD) is included in IgG4RD [10]. The main features of MD are high serum IgG4 and bilateral lacrimal/salivary persistent swelling [10]. A case of MD with eosinophilic granulomatosis with polyangitis (Churg-Strauss syndrome) was previously reported with coexistence of neuropathy due to allergic reaction [11]. On the other hand, a case of neuropathy with MD itself has not been reported, even if a lot of other IgG4RD were reported to coexist with MD.

The clinical course of IgG4RD is diverse. Exacerbation is often seen during tapering of the steroid dose [12], while spontaneous remission is seen in some cases [13]. Clinical outcomes of peripheral neuropathy are unclear, in contrast to other IgG4RDs, such as autoimmune pancreatitis (AIP) [14] and IgG4-related sclerosing cholangitis (IgG4-SC) [15].

Here, we report the clinical course of a case of MD with mononeuritis multiplex.

\section{Case report}

An 82-year-old Japanese man was admitted to our hospital with gait disturbance. He had previously been diagnosed with angina, atrial fibrillation, hypertension and diabetes mellitus (DM). He had experienced pain and swelling of the left hand and right toe a few days before admission. These symptoms were progressive and made it difficult for him to walk. Similar symptoms had appeared on the right upper and lower extremities one year before, and he was treated with an antibiotic at another hospital. However, the pain and swelling had not improved, and joint deformation remained.

On admission, his blood pressure, heart rate and temperature were $150 / 100 \mathrm{mmHg}, 92$ beats per minute, and $37.0^{\circ} \mathrm{C}$. His consciousness state was alert. Both upper eyelids, the salivary gland, the dorsum of the left hand and both legs were swollen (Fig. 1A-1, 2). He also reported joint pain and showed a limited range of motion. Scratches were also seen on both dorsa of the feet. Neurologi-

Correspondence: Atsushi Mizuma, MD, PhD, Department of Neurology, Tokai University School of Medicine, 143 Shimokasuya, Isehara,

Kanagawa, 259-1193, Japan, e-mail: a-mizuma@is.icc.u-tokai.ac.jp

Submitted: 14.07.2016; Accepted: 20.10.2016 
cal examination showed motor weakness of distal limbs (manual muscle testing 3/5) and decreased touch, pain and vibration sensation of the dorsum of the left hand and both legs. Deep tendon reflex in both legs was also decreased. Laboratory examinations revealed high inflammatory re-

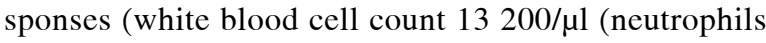
$72.2 \%$, lymphocytes $16.7 \%$, eosinophils $0.1 \%$ ), C-reactive protein $14.37 \mathrm{mg} / \mathrm{dl}$, elevated sedimentation rate $34 \mathrm{~mm} / \mathrm{h}$ ) and hyperuricemia $(9.7 \mathrm{mg} / \mathrm{dl})$. Liver function (aspartate transaminase, alanine transaminase, $\gamma$-glutamyltransferase, lactate dehydrogenase), renal function (blood urea nitrogen, creatinine), and amylase were within normal ranges. Glycometabolism $\left(\mathrm{HbA}_{1 \mathrm{c}}[5.7 \%]\right)$, thyroidal function (thyroid-stimulating hormone, free T4), creatine kinase, and gonadal hormones (testosterone [0.69 $\mathrm{ng} / \mathrm{ml}]$ )were also within normal ranges. High serum $\operatorname{IgG}(2417 \mathrm{mg} / \mathrm{ml})$ and $\mathrm{IgG} 4(630 \mathrm{mg} / \mathrm{dl}, 26.1 \%$ of total $\mathrm{IgG})$ were seen, although autoimmune antibodies were all negative (rheumatoid factor, anti-nuclear antibody, anti-cyclic citrullinated peptide antibody, anti-topoisomerase antibody, anti-centromere antibody, anti-SS-A antibody, anti-SS-B antibody, and anti-neutrophil cytoplasmic antibody). As for other serum laboratory examinations, immuno-electrophoresis showed a chronic inflammatory pattern and serum vascular endothelial growth factor was elevated $(351 \mathrm{pg} / \mathrm{ml})$. Cerebrospinal fluid (CSF) showed no elevation of protein or pleocytosis. Urinalysis was negative for protein, occult blood, glucose, and ketones. The cultivation test revealed Staphylococcus aureus in two blood cultures and no bacterium in CSF. No abnormal lesions were seen in brain and spine MRI. Wrist MRI revealed swelling of the synovium and myelin sheath with fluid accumulation on the left side. The electromyographic (EMG) study showed signs of mononeuritis multiplex (Table 1). In conduction studies, compound muscle action potentials (CMAPs) and sensory nerve action potentials (SNAPs) were absent in the left median and ulnar nerves. In the right median and left lower extremities, CMAP amplitudes were reduced. Needle EMG showed neurogenic changes with the voluntary movement of the upper and lower extremities, and no denervation potential at rest. Enhanced computed tomography and gallium scintigraphy showed no inflammatory lesions or malignant tumors. Lymph node echography showed only reactive swellings. Lacrimal and salivary gland echography showed low echoic mass lesions, suggesting swelling of the glandular tissue. Based on this finding, a la-
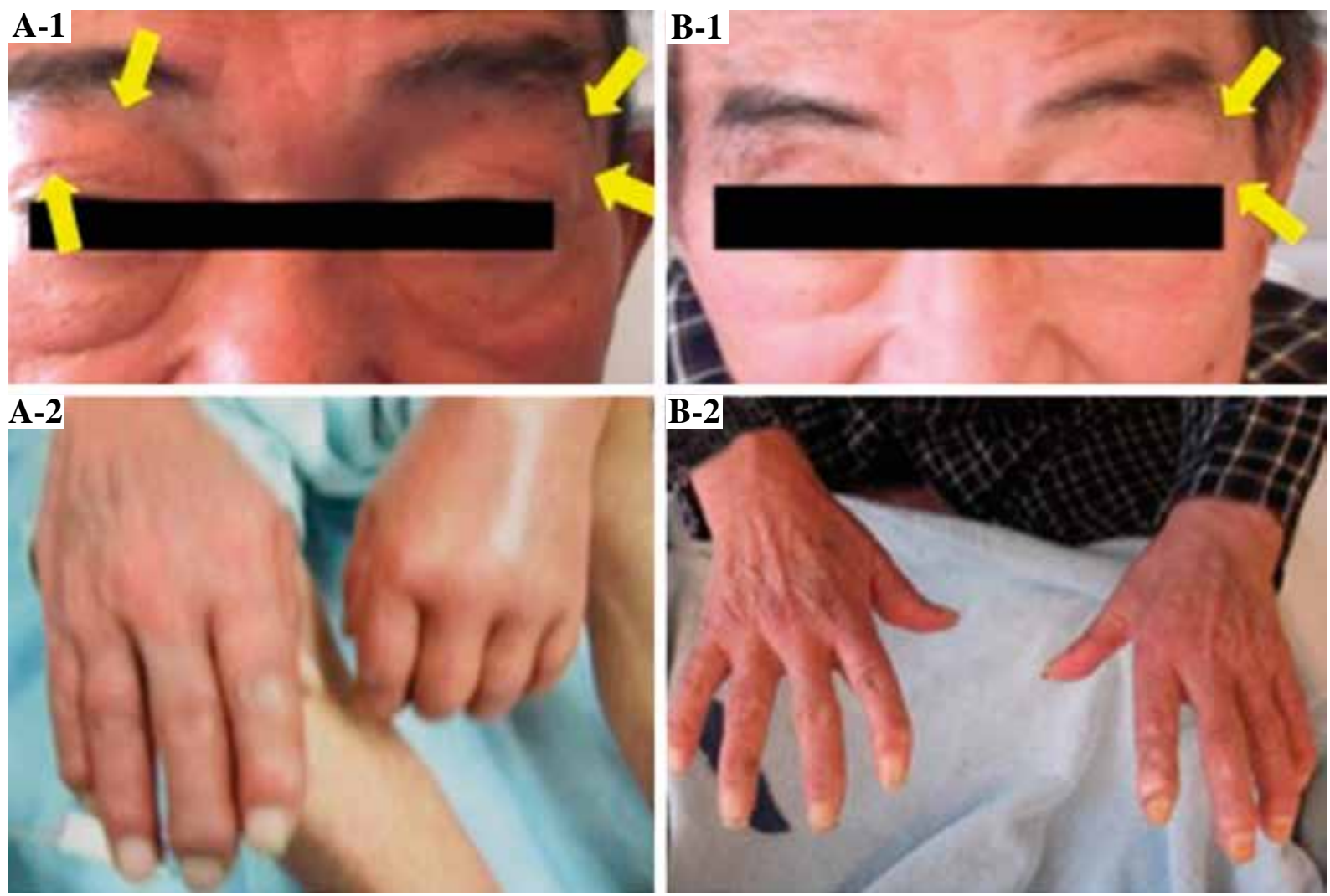

Fig. 1. A) On admission. Both upper eyelids (1) and the left dorsum of the hand (2) were swollen. Joint pain and limited range of motion were also present. B) After steroid therapy. Swelling of upper eyelid (1) and left dorsum of the hand (2) were improved. Joint pain had disappeared and range of motion was improved 
Table 1. Nerve conduction study showed signs of mononeuritis multiplex. Compound muscle action potentials (CMAPs) and sensory nerve action potentials (SNAPs) were absent in the left median and left ulnar nerves, respectively

\begin{tabular}{lcccc}
\hline & Site & Latency $(\mathbf{m s})$ & Amplitude $(\mathbf{m V})$ & Nerve conduction velocity $(\mathbf{m} / \mathbf{s})$ \\
\hline $\begin{array}{l}\text { Motor } \\
\text { conduction } \\
\text { study }\end{array}$ & Left median & Not detected & Not detected & Not detected \\
\cline { 2 - 5 } & Right median & 4.32 & 1.826 & 43.6 \\
\cline { 2 - 5 } & Left ulnar & 3.99 & 3.299 & 41.7 \\
\cline { 2 - 5 } & Left tibial & 4.59 & Not detected & Not detected \\
\cline { 2 - 5 } & $\begin{array}{c}\text { Left } \\
\text { peroneal }\end{array}$ & Not detected & Not detected & Not detected \\
\hline $\begin{array}{l}\text { Sensory conduction } \\
\text { study }\end{array}$ & Left ulnar & Not detected & & \\
\hline
\end{tabular}
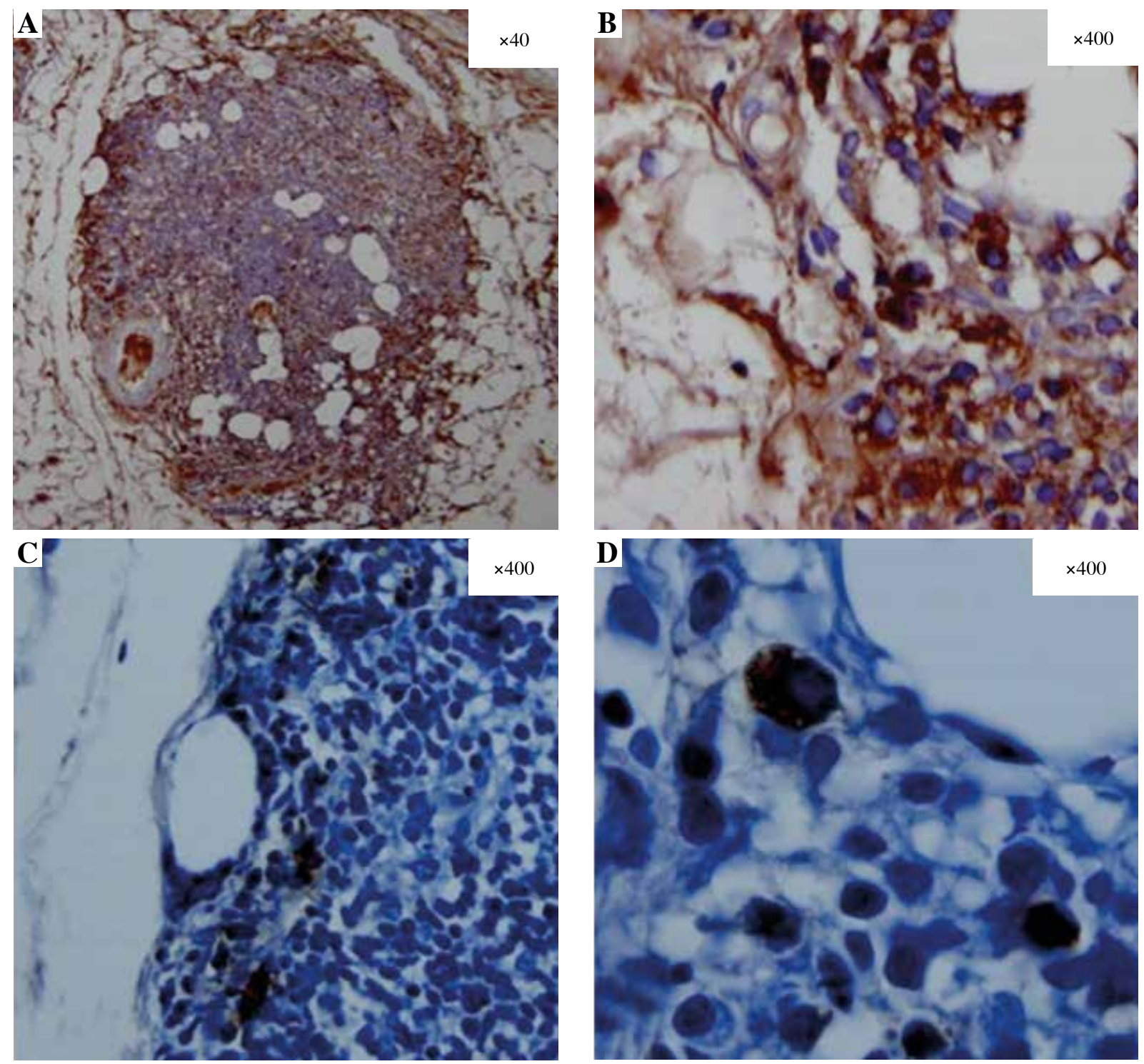

Fig. 2. A, B) Histopathologic examination of lacrimal gland biopsy revealed lymphoid follicles with germinal center. IgG-positive plasma cells on the lymphoid follicles were visualized by enzyme immunostaining. C, D) Enzyme immunostaining with an anti-IgG4 antibody revealed IgG-positive plasma cells, accounting for about a half of IgG4-positive cells on the lymphoid follicles 
crimal gland biopsy was performed and showed lymphoid follicles with a germinal center. Enzyme immunostaining with anti-IgG4 antibody revealed IgG-positive plasma cells, of which about a half were IgG4-positive, on the lymphoid follicles (Fig. 2). Based on these results, Mikulicz's disease was diagnosed.

\section{Clinical course}

The clinical course is shown in Fig. 3. On admission, antibiotic therapy (cefazolin $2 \mathrm{~g} /$ day) was started to treat cellulitis, based on the results of blood culture. Considering comorbid polyarthritis with hyperuricemia, non-steroidal anti-inflammatory drugs (loxoprofen $180 \mathrm{mg} /$ day) and benzbromarone were also started. Although his joint pain and swelling were reduced, the inflammatory reaction and neuropathy remained. The subsequent laboratory finding of high serum IgG4 and the lacrimal gland swelling were suggestive of IgG4RD. Nerve biopsy was not performed because of the remaining ankle swelling and pain. After lacrimal gland biopsy, he was diagnosed on MD and oral prednisolone was started at $40 \mathrm{mg} / \mathrm{day}$, and the clinical symptoms (Fig. 1B-1, 2) and EMG findings improved. After discharge, the steroid dose was tapered because of his DM, reaching $3 \mathrm{mg}$ /day after two years. However, at that time, lacrimal gland swelling reappeared, together with gait disturbance and elevation of serum IgG4. The steroid dose was increased to $10 \mathrm{mg} /$ day and these symptoms improved.

\section{Discussion}

We present a case of recurrent mononeuritis multiplex with MD. This is of interest, because the frequency of peripheral neuropathy associated with IgG4RD remains unclear. Also, the recurrence of symptoms during tapering of steroid therapy suggests that addition of an immunosuppressant might be effective to prevent relapse.

Reported forms of IgG4RD include hypophysitis, pachymeningitis [7, 8], and cranial nerve neuropathy [16]. However, few cases with peripheral neuropathy in the distal extremities have been reported. Ohyama et al. [9] reported IgG4-related peripheral neuropathy diagnosed by sural nerve biopsy. Decrease of myelinated fibers, axonopathy, and infiltration of IgG4-positive plasma cells on the epineurium were pathologically recognized [9]. This report suggested that $\mathrm{IgG} 4$ related neuropathy was caused by vasculitis based on the pathological findings. As for the other etiology of IgG4-related neuropathy, Yamamoto et al. [11] reported a case of eosinophilic granulomatosis with polyangitis (Churg-Strauss syndrome) that met the diagnostic criteria for MD. This report suggested an association between IgG4-related neuropathy and vasculitis due to allergic reaction. The major difference in our case was occurrence of vasculitis due to MD rather than another

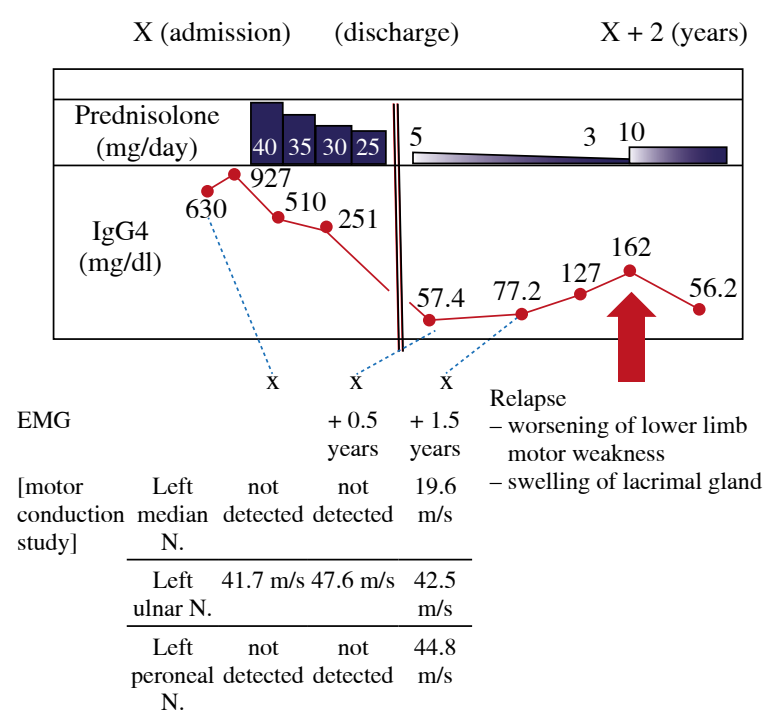

Fig. 3. On admission, antibiotic therapy (cefazolin 2 g/day) was started to treat cellulitis. Non-steroidal anti-inflammatory drugs (loxoprofen $180 \mathrm{mg} /$ day) and benzbromarone were also started to treat polyarthritis with hyperuricemia. After a diagnosis of IgG4 related disorder, oral prednisolone was started at $40 \mathrm{mg} /$ day. After starting oral prednisolone, the clinical symptoms and nerve conduction study findings improved. After discharge, the steroid dose was tapered. But at the time of reducing $3 \mathrm{mg} /$ day of prednisolone dose, both lacrimal gland swelling and gait disturbance reappeared. Serum IgG4 was also elevated. The steroid dose was increased to $10 \mathrm{mg} /$ day and these symptoms improved

allergic reaction, because there was no history of allergic disease and eosinophilia. Also, we needed to consider about the correlation with cellulitis from scratches and DM.

Aside from the neuropathy, we firstly considered that the limb swelling with joint pain was caused by cellulitis from scratches and started antibiotic therapy, even if we also considered it to be due to polyarthritis with hyperuricemia. After starting therapy, these symptoms were reduced, except for the motor weakness and sensory disturbance. Also, the limb swelling with joint pain was not in parallel with lacrimal gland swelling and neuropathy throughout the entire course. Therefore, we concluded that the cause of the limb swelling and joint pain was cellulitis. On the other hand, this cellulitis was not considered to be the cause of mononeuritis multiplex, but it was also considered to be important as an initial trigger of immunological inflammation. Secondly, we considered the correlation between mononeuritis multiplex and DM. Although the patient had a history of DM, other complications were not detected and the control of glycometabolism was good without using anti-diabetic drugs. Furthermore, this neuropathy was in parallel with serum IgG4 levels and the status 
of MD (lacrimal gland swelling). From the above consideration, we finally made the diagnosis of mononeuritis multiplex associated with IgG4RD, due to the concurrence of IgG4 and Mikulicz's disease, even if sural nerve biopsy could not be performed because of ankle edema and pain.

Steroid maintenance therapy of AIP was reported to be effective in terms of remission rate and recurrence rate in a multicenter trial [14]. The international consensus recommendation is $0.6-1.0 \mathrm{mg} / \mathrm{kg}$ of oral prednisolone as remission induction therapy and $5 \mathrm{mg} /$ day of prednisolone as maintenance therapy [17]. The recurrence rate without maintenance steroid therapy was reported to be about $50 \%$ [15], and maintenance steroid therapy is also recommended in other reports $[12,14]$. On the other hand, the risk factors for recurrence appear to be different for each IgG4targeted organ. AIP of pancreatic bile duct lesions, duration of high IgG4 [13, 14, 18] lacrimal gland sialadenitis [19] of juvenile onset and male sex were reported as risk factors for recurrence.

Recurrence has been reported at a rate of $15-56 \%$ on stopping steroid therapy or reducing it to $5-20 \mathrm{mg} /$ day [13, $14,20,21]$. It is suggested that an immunosuppressant (azathioprine, rituximab, or cyclosporine) should be added $[22,23]$ or the steroid dose should be increased at the time of recurrence [14]. In our case, recurrence was seen when the steroid dose was reduced because of the patient's DM. Increase of the steroid dose from 3 to $10 \mathrm{mg} /$ day was effective to treat the relapse in our patient.

In conclusion, we reported a case of recurrent mononeuritis multiplex with MD. In this case, steroid therapy was effective for recurrent IgG4RD neuropathy, but in general, a suitable combination of a maintenance dose of steroid and an immunosuppressant may be desirable to prevent relapse of IgG4RD.

\section{The authors declare no conflict of interest.}

\section{References}

1. Hamano H, Kawa S, Horiuchi A, et al. (2001): High serum IgG4 concentrations in patients with sclerosing pancreatitis. N Engl J Med 344: 732-738.

2. Umehara H, Okazaki K, Masaki Y, et al. (2012): Research Program for Intractable Disease by Ministry of Health, Labor and Welfare (MHLW) Japan G4 team. A novel clinical entity, IgG4-related disease (IgG4RD): general concept and details. Mod Rheumatol 22: 1-14.

3. Okazaki K, Uchida K, Ohana M, et al. (2000): Autoimmune-related pancreatitis is associated with autoantibodies and a Th1/Th2-type cellular immune response. Gastroenterology 118: 573-581.

4. Umehara H, Okazaki K, Masaki Y, et al. (2012): Comprehensive diagnostic criteria for IgG4-related disease (IgG4-RD), 2011. Mod Rheumatol 22: 21-30.

5. Kamisawa T, Okamoto A (2006): Autoimmune pancreatitis: proposal of IgG4-related sclerosing disease. J Gastroenterol 41: 613-625.

6. Kamisawa T, Zen Y, Pillai S, Stone JH (2015): IgG4-related disease. Lancet 385: 1460-1471.
7. Kupersmith MJ, Martin V, Heller G, et al. (2004): Idiopathic hypertrophyic pachymeningitis. Neurology 62: 686-694.

8. Shimatsu A, Oki Y, Fujisawa I, Sano T (2009): Pituitary and stalk lesions (infundibulo-hypophysitis) associated with immune G4-related systemic disease: an emerging clinical entity. Endocr J 56: 1033-1041.

9. Ohyama K, Koike H, Iijima M, et al. (2013): IgG4-related neuropathy: a case report. JAMA Neurol 70: 502-505.

10. Yamamoto M, Takahashi H, Ohara M, et al. (2006): A new conceptualization for Mikulicz's disease as an IgG4-related plasmacytic disease. Mod Rheumatol 16: 335-340.

11. Yamamoto M, Takahashi H, Suzuki C, et al. (2010): Analysis of serum IgG subclasses in Churg-Strauss syndrome - the meaning of elevated serum levels of IgG4. Intern Med 49: 1365-1370.

12. Yamamoto M, Takahashi H, Shinomura Y (2011): Mikulicz's disease and the extraglandular lesions. Curr Immunol Rev 7: 162-171.

13. Kawa S, Okazaki K, Kamisawa T, et al. (2014): Amendment of the Japanese Consensus Guidelines for Autoimmune Pancreatitis, 2013 III. Treatment and prognosis of autoimmune pancreatitis. J Gastroenterol 49: 961-970.

14. Kamisawa T, Okazaki K, Kawa S, et al. (2009): Working Committee of the Japan Pancreas Society and the Research Committee for Intractable Pancreatic Disease supported by the Ministry of Health, Labour and Welfare of Japan. Standard steroid treatment for autoimmune pancreatitis. Gut 58: 1504-1507.

15. Ghazale A, Chari ST, Zhang L, et al. (2008): Immunogloblin G4-associated cholangitis: clinical profile and response to therapy. Gastroenterology 134: 706-715.

16. Sogabe Y, Ohshima K, Azumi A, et al. (2014): Location and frequency of lesions in patients with IgG4-related ophthalmic diseases. Graefes Arch Clin Exp Ophthalmol 252: 531-538.

17. Shimosegawa T, Okazaki K, Kamisawa T, et al. (2011): International consensus diagnostic criteria for autoimmune pancreatitis. Guidelines of the International Association of Pancreatology. Pancreas 40: 352-358.

18. Raina A, Yadav D, Krasinskas AM, et al. (2009): Evaluation and management of autoimmune pancreatitis: experience at a large US center. Am J Gastroenterol 104: 2295-2306.

19. Yamamoto M, Nojima M, Takahashi H, et al. (2015): Identification of relapse predictors in IgG4-related disease using multivariate analysis of clinical data at the first visit and initial treatment. Rheumatology(Oxford) 54: 45-49.

20. Saeki T, Kawano M, Mizushima I, et al. (2013): The clinical course of patients with IgG4-related kidney disease. Kidney Int 84: 826-833.

21. Hagiya C, Tsuboi H, Yokosawa M, et al. (2014): Clinicopathological features of IgG4-related disease complicated with orbital involvement. Mod Rheumatol 24: 471-476.

22. Hart PA, Topazian MD, Witzig TE, et al. (2013): Treatment of relapsing autoimmune pancreatitis with immunomodulators and rituximab: the Mayo Clinic experience. Gut 62: 16071615.

23. Khosroshahi A, Carruthers MN, Deshpande V, et al. (2012): Rituximab for the treatment of IgG4-related disease: lessons from 10 consecutive patients. Medicine (Baltimore) 91: 57-66. 